\title{
A Rudimentary History of Dynamics
}

\author{
Andrew Ross \\ Norwegian Marine Technology Research Institute (MARINTEK), Marine Technology Centre, NO-7450 Trondheim, \\ Norway. E-mail: andrew.ross@marintek.sintef.no

\begin{abstract}
This article presents a condensed overview of the development of dynamics from Ancient Greece through to the late 18th century. Its purpose is to bring an often neglected topic to the control community in as interesting a fashion as the author can achieve.
\end{abstract}

Keywords: History of science, dynamics, mechanics, kinematics, Newtonian equations, Newton's Laws

\section{Introduction}

This article describes a few key points in the development of the field of dynamics. It begins with a brief overview of dynamics' origins in Ancient Greece, and progresses through the Middle-Ages and the Renaissance, stopping at the times, places, and people that the author considers most relevant. Following that, it summarises the appearance of the Principia, and proceeds to explain its development into what we now call Classical Mechanics.

It should be clear that the contents are not new: they are well known in many fields, and have been for many years. They are presented to the control community in the hope that knowledge of, and interest in, the history of science might be improved. Any contribution is solely in bringing the subject to an audience that is perhaps generally unacquainted with it. Not only are the contents not new, they are also by no means complete or comprehensive. With those caveats, the author hopes that some of the intended audience might find value in what is presented.

Take what the pre-eminent physicist and philosopher of science, Ernst Mach, wrote of Newton's contributions (Mach, 1886):

Newton discovered universal gravitation and completed the formal enunciation of the mechanical principles now generally accepted. Since his time no essentially new principle has been stated. All that has been accomplished in mechanics since his day has been a deductive, formal, and mathematical development of mechanics on the basis of Newton's Laws. ${ }^{1}$

The sentiments expressed by Mach are broadly in line with the opinions of many in academia. The popularity of a belief does not allow us to conclude whether it is factual or not.

The motivation for writing this paper stems from the sentiments of the great physicist, mathematician, historian of science, and polemicist, Clifford Truesdell $^{2}$. In his essay A Program Toward Rediscovering the Rational Mechanics of the Age of Reason(Truesdell, 1968), he wrote:

The scientists, in so far as they take any note of history at all, not only have shared the historians' neglect of the later [i.e., after Newton] mathematical development of mechanics but also, in the main, have ignored what the historians have learned about the earlier periods and have rested content with Mach's whole view or a rudimentary abstract of it.

\footnotetext{
${ }^{1}$ That general relativity has since superseded classical mechanics is of no importance here.

${ }^{2}$ The term "natural philosopher" ought to suffice, but unfortunately has not enjoyed popular or correct usage in recent times (Noll, 1996).
} 
Whether reading a textbook on robotics, marine engineering, aerospace engineering or, indeed, on mechanics itself, the statement above is too often applicable. Discussions of dynamics almost invariably begin by citing the work of Newton (1687) in his Principia, and seldom proceed further than this opus. It is as if classical mechanics arose from the genius of Newton alone.

This hagiography does a great disservice to at least four parties: firstly, to the scholars whose works predate Newton; secondly, to Newton's contemporaries and successors, who actually synthesised the dynamics that we know and apply today; thirdly, to the presentday students who desire an accurate history of dynamics; and fourthly to Newton himself, whose memory is sullied by the misrepresentation of his work.

This neglect leads us to question why this history is being contemplated here. The problem is that the history of science, a large and growing field, seems to filter little of its knowledge to the practitioners of science. Scientific careers can be built on advanced topics with absolutely no concept of what lies in the foundations. The history of a science is vital to a humble understanding of that science.

Awkwardly, the history of a science can only really be grasped and analysed after the subject itself is sufficiently well understood. However, once that science is understood and a university degree comes to its end, there is no drive to put the learning into a historical perspective. A modern engineering degree is then more like an advanced tradeschool diploma than the higher form of learning and understanding that it ought to be, and that it used to be.

A cursory and often inaccurate glance at history seems sufficient nowadays, but should it be? The development of dynamics is a much longer, halting and laborious story that neither began nor ended with Isaac Newton.

\section{Dynamics in Antiquity}

An analysis of motion ought to begin with the Ancient Greeks $^{3}$. Since the influence of the Greeks lasted two millennia, it is inconceivable to describe the growth of dynamics without mentioning them.

The dominant figure in the ancient development of dynamics was Aristotle (384 BC- 322 BC). His writings (Aristotle, 330 B.C.) on this and on many other subjects held sway over much of science for the next two thousand years. Much of his reasoning on motion

\footnotetext{
${ }^{3}$ Greek philosophy is generally taken to begin with the work of Thales of Miletus (ca. 624 BC-ca. 546 BC), in modern-day Turkey.
}

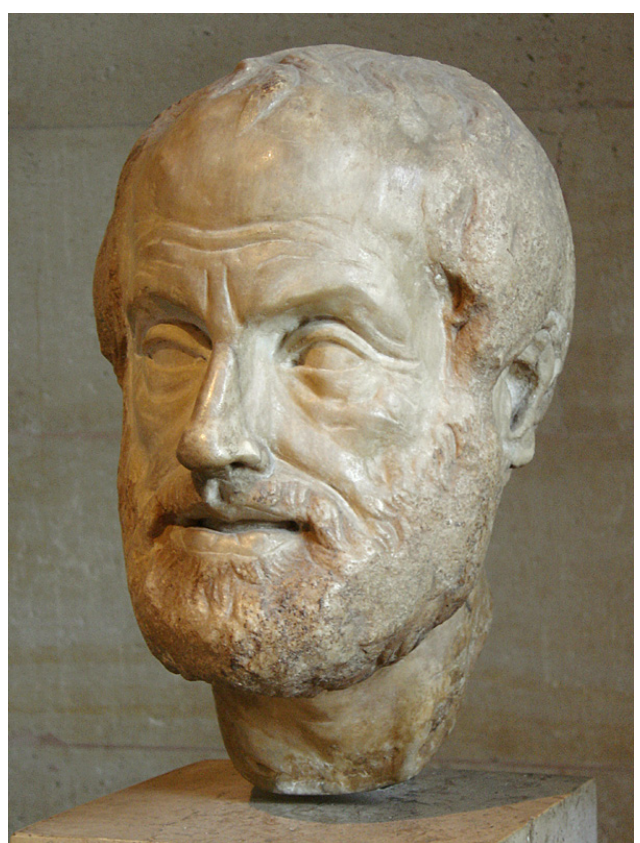

Figure 1: Replica of Lysippos' Aristotle.

stemmed from the faulty concept of the classical elements (fire, air, water and earth). Each of these is given its own natural place in the world: fire at the top; air underneath fire; water below air; and finally earth resting beneath them all.

Whenever an element was taken from its natural place it would endeavour to return. This reasoning explained why an air bubble breathed underwater floats to the surface, and why a rock thrown upwards falls back to the Earth. Each object was then a combination of all of these. A feather, lighter than a rock, must have more air than the rock, but less than the air itself. From this line of thinking arose "natural motion": motion that occurs due to the nature of the object. All other motion was violent; it had a separate cause. A brick falling to the ground would be natural, but a brick thrown through the air would be violent.

Aristotle concluded that heavier objects fall faster than light objects, and that this fall-rate is proportional to their weights: an object twice as heavy falls twice as fast. He also reasoned that the speed of progression through a medium was inversely proportional to the density of that medium. This reasoning implied that the speed of progression in the void would be infinite; thus he concluded that the very existence of a void was impossible (Aristotle (330 B.C.), Book IV:8).

...between any two movements there is a ratio (for they occupy time, and there is a ratio between any two times, so long as both 
are finite), but there is no ratio of void to full.

In the same section, he wrote that if a void were to exist, heavy objects would fall at the same rate as light ones ("Therefore all will possess equal velocities. But this is impossible."). He used this supposed equality of fall rates to then say by modus tollens that a void cannot exist. He further wrote that, in a void, there would be no reason for a body to stay in one place or move to another, and so motion would continue forever. It is often said, based on this statement, that he enunciated or foresaw a principle of inertia, but this is only possible by a selective reading of his works.

Among the various physical questions pondered by ancient philosophers, the question of why an arrow continues to fly after it has left its bowstring was particularly perplexing. Aristotle reasoned that the arrow displaced the air in front of it, which rushed behind and then pushed the arrow forwards. The idea of a thing moving violently without some other thing pushing it along the way; moving without a mover, was entirely alien to Aristotelians. This fallacious separation of natural and violent motion would haunt physics for two thousand more years.

The progress towards a truer representation was slow and halting. Aristotle's worldview became ingrained upon both Western and Arabic science and theology. His prevalence in the latter of these fields impacted the progression of the former. Much of it became Church dogma. By raising his theology above and beyond criticism, it raised a protective wall, by proxy, around his physics.

The lengthy dominance of Aristotle is now difficult to imagine. Even into the early Renaissance entire contributions on physics from philosophers would consist solely of commentaries on Aristotle's works: two millennia after they were composed.

The 6th century Alexandrian philosopher, John Philoponus (ca. 490-ca. 570), wrote extensive critiques of Aristotelian physics (Philoponus, 2006), and it is here that the inklings of a modern approach to dynamics can be seen.

Philoponus found little satisfaction in Aristotle's approach to motion, indeed he also found little satisfaction in his other approaches. In his commentaries he demolished Aristotle's work on both natural and violent motion. For natural motion, Philoponus posited that an object has a natural rate of fall. Falling through a medium would hinder this natural rate:

But a certain additional time is required because of the interference of the medium.

He introduced a natural fall rate in the void, and subtracted from this the effect of the resistance of the medium. This concept allowed him to reject the Aristotelian concept that the speeds at which objects fall at are in proportion to their weights. He did this with appeal to the same kind of experiment carried out in Renaissance Italy around a millennium later ${ }^{4}$. Philoponus did not believe in the equality of fall-rates in the void. In fact he concluded that this concept was wrong. His belief was that heavier objects $d o$ fall faster than light ones in a void.

For violent motion, he asserted that when an object is moved, it is given a finite supply of forcing impetus ${ }^{5}$ : a supply of force that, while it lasted, would explain the object's continuing motion:

Rather it is necessary to assume that some incorporeal motive enèrgeia is imparted by the projector to the projectile...

This incorporeal motive enèrgeia is exhausted over the course of an object's motion, which rests once this exhaustion is complete. This property was internal to the body. He struck fairly close to some kind of rudimentary concept of kinetic energy. At the very least, he struck close to some concept which we can now relate to kinetic energy.

The conclusion of the sentence quoted above is:

....and that the air set in motion contributes either nothing at all or else very little to this motion of the projectile.

The strongest and most groundbreaking insight that Philoponus made was that a medium does not play a role in maintaining motion. It acts as a retarding force. This notion was in direct opposition to Aristotle, who required that the medium should cause the continuing motion. This paradigm shift that John Philoponus introduced allowed him to explain that motion in a void was possible. His lasting contribution is with these qualitative analyses. His quantitative explanations are without merit, although these analyses resonate through Galileo's dynamics.

In the centuries that followed Philoponus, other philosophers followed in a staggering and haphazard progression towards Newton. It would be another millennium before Aristotelian motion would be disregarded. The reasons are various, but much of them are theological in nature. Philoponus' writings on Tritheism were declared anathema by the Church, which led to the neglect, condemnation, and ridicule of his writings. Zimmerman had the following to say (Zimmerman, 1987):

\footnotetext{
${ }^{4}$ The comparison of the speeds of falling objects, carried out by Benedetti around 1553 (commonly attributed to Galileo).

${ }^{5}$ Impetus theory, the precursor to the modern principle of inertia, can properly be attributed to Philoponus.
} 
His writings, then and later, enjoyed notoriety rather than authority.

The inferior works on mechanics from his contemporaries, such as Simplicius, were treated in a more favourable light.

\section{The Middle Ages}

In the following centuries, the development of dynamics was very slight. There is a pernicious popular belief that science stood still from the fall of the Western Roman Empire (476 A.D.) until the Renaissance: the so called Dark Ages. While the remark may hold water for certain periods of the Early Middle Ages, it has no standing whatsoever with the High and Late Middle Ages. The idea that the world of understanding stood still for a millennium is a hopelessly incorrect one.

Aristotle's views, or variations on these, were analysed further by the likes of the Andalusian-Arabs Avempace and Averröes ${ }^{6}$ in the mid-13th century. The gratitude owed to these philosophers should not be understated. It is through their works that Philoponus' thoughts were preserved: his books were not published in Western Europe until the early 16th century. Averröes wrote such extensive treatises on Aristotelian physics and theology that he was nicknamed The Commentator by Thomas Aquinas. The intellectual stupor existed in the West because an Aristotelian theological worldview was dogma. Those studying mechanics were reticent to go further than simple reinterpretation of Aristotle, even when so much of it was clearly wrong.

The stimulus that reinvigorated the field can be traced to the Condemnations of 1277. In this year, Tempier, the Bishop of Paris, condemned various doctrines enveloping much of radical Aristoelianism and Averröeism, among others. This event is important because the condemnation of Aristotle's theology led philosophers to question the truth of the rest of his worldview. Deviating from dogma was then, and remained for centuries more, very dangerous for philosophers, but now Aristotle's physics were no longer protected. The importance of the Condemnations led to what Duhem (1917) called:

...a large movement that liberated Christian thought from the shackles of Peripatetic and Neoplatonic philosophy and produced what the Renaissance archaically called the science of the 'Moderns.'

\footnotetext{
${ }^{6}$ Ibn Bājjah and Ibn Rushd in Anglicised Arabic respectively.
}

Soon after, in the early 14th century, the Oxford Calculators ${ }^{7}$ explained, in a kinematic sense, the motion of objects under uniform acceleration. Importantly, these men did not concentrate solely on the qualitative description of motion. What was previously a murky description of motion became a quantitative derivation. They answered kinematic questions numerically. What is fantastic is that the notion of instantaneous speed was within their grasp, even without the strong grip afforded us by calculus. The mean-speed theorem dates from this period, and is attributed to William Heytesbury $^{8}$. That theorem sprung from the investigations into how two bodies moving along a path at different speeds might arrive at an endpoint at the same time (see the essay "Laws of Motion in Medieval Physics" in Moody (1975)). They were additionally responsible for separating motion itself from its causes: the separation of kinematics and kinetics. Bradwardine ${ }^{9}$ also noted:

All mixed bodies ${ }^{10}$ of similar composition will move at equal speeds in a vacuum.

The statement above shows that the Mertonians were well aware of the principle that objects of the same composition fall at the same rate, regardless of their mass. The fall rates were still explained in terms of the nonsense classical elements of Ancient Greece, but they were explained. Within their work can be found thorough analyses of uniform and accelerated motion. Their analytical approaches to motion were well received Europe-wide.

French priest Jean Buridan (1300-1358) was by most accounts the giant of fourteenth century philosopy. He expounded a theory that can properly be described as an early and rudimentary concept of what we now call inertia.

He posited in a similar manner to Philoponus that the motion of an object was internal to it, and importantly recognised that this impetus does not dissipate through its own motion: that something else must act upon the object to slow its motion. His insights into the implications of this were more advanced than anything prior. In discussing a thrown projectile, he said that it would:

...continue to be moved as long as the impetus remained stronger than the resistance,

\footnotetext{
${ }^{7}$ The Oxford Calculators were a group of 14 th century academics based at Merton College, Oxford, and included William Heytesbury, Thomas Bradwardine, Richard Swineshead and John Dumbleton.

${ }^{8}$ Bizarrely attributed to Galileo by many.

${ }^{9}$ The selfsame Bradwardine spoken of in Chaucer's Canterbury Tales.

${ }^{10} \mathrm{~A}$ mixed body is one that consists of two or more of the classical elements: fire, air, water and earth.
} 


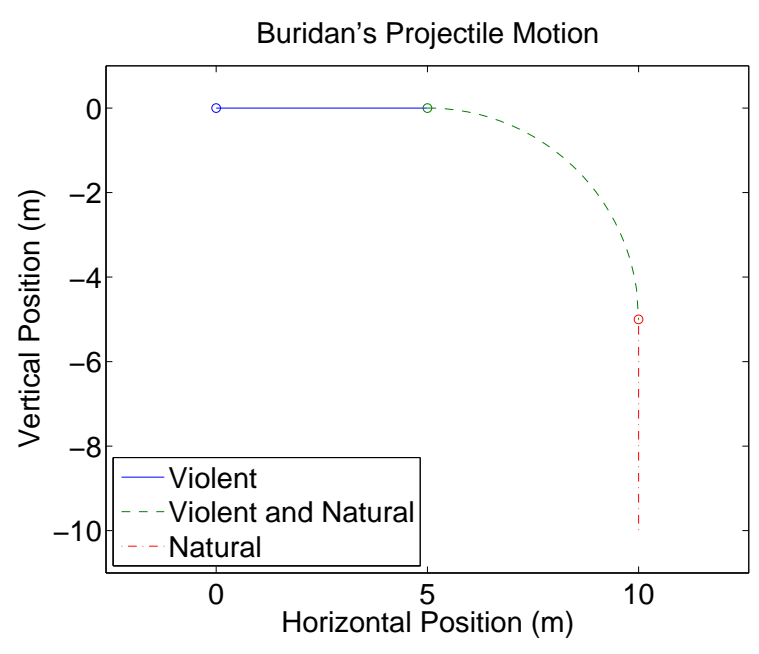

Figure 2: Albert of Saxony's motions.

and would be of infinite duration were it not diminished and corrupted by a contrary force resisting it or by something inclining it to a contrary motion.

His statement is an early and rudimentary notion that is qualitatively similar to Newton's First Law. He entertained this notion of infinite motion, a full three centuries before. His talent in descriptions of the qualitative properties was not matched by his talent in the quantitative.

Buridan's student, Nicolo Oresmè (ca. 1323-1382), developed geometrical descriptions of motion. More than that, he used geometry as a method of explaining the variations of any physical quantity. As great as this was, he had a poorer understanding of dynamics than his tutor, and treated impetus as something which decays with motion (Wallace, 1981). Oresmè's work is a prime example of the stumbling advancement of dynamics: it was rare that any one person could advance in all areas at once.

Albert of Saxony (ca. 1316-1390), another student of Buridan, took impetus theory forwards in projectile motion. For an object propelled horizontally, he reasoned that the motion had three distinct periods. The first of these was purely horizontal, where the body moved by its own impetus. The second was a curve towards the ground, as gravity began to take effect. The third was a vertical drop, as gravity took over and impetus died. Although maintaining the distinction between natural and violent motion, Albert at least came closer to the true shape of projectile motion.

It is quite difficult to conceive the true effect that the philosophers from the Oxford and Parisian schools had on mechanics, and on science in general. Mechanics had moved from indistinct qualities into defined quantities: if an object moves at this speed, how far does it go in this amount of time? If an object accelerates in this manner, what will its speed be after a given period? These questions were asked and answered.

Shortly after Giovannia di Casalè (d. ca. 1375) returned to Genoa from studying at Oxbridge, he developed a geometric approach in his book "On the velocity of the motion of alteration" similar to that of Oresmè. This work influenced the Venetian, Giambattista Benedetti, in his 1553 demonstration of the equality of fall-rates. The influence that Casali's geometric approach wielded is evident while reading Galileo's works on kinematics.

An important point is then evident: the field of kinematics had leapt ahead of dynamics. Truesdell (1968) speaks of the impact of the Calculators in the following glowing terms:

In principle, the qualities of Greek physics were replaced, at least for motions, by the numerical quantities that have ruled Western science ever since.

While kinematics was becoming more and more capable of describing both uniform and accelerated motion, and was able to quantify these analytically, numerically and geometrically, philosophers remained unable to explain the why behind them. The causes of motion, now separate and distinct from kinematics, were not very much closer to being discovered. This situation changed very little until the late 16 th century.

\section{Galileo}

Galileo Galilei (1564-1642), shown in Figure 3, sought these causative descriptions of motion: he was the first of the modern dynamicists. The Italian was well-read in the workings of both the Parisian and Mertonian schools. From these, he set out into the still poorly understood field of kinetics.

To move forward, he examined the most successful of the ancient sciences: Archimedes' hydrostatics. He took those principles as inspiration to examine the motion of a falling object. He utilised no mixed-body theory of matter. Instead, he treated bodies, and the media through which they travel, in terms of their densities $^{11}$.

Archimedes' propositions explain the forces of buoyancy in equilibrium: they detail where an object will rest in a body of water. Galileo extended these principles from static into dynamic concepts. Archimedes

\footnotetext{
$\overline{11}$ or, rather, their specific gravity.
} 


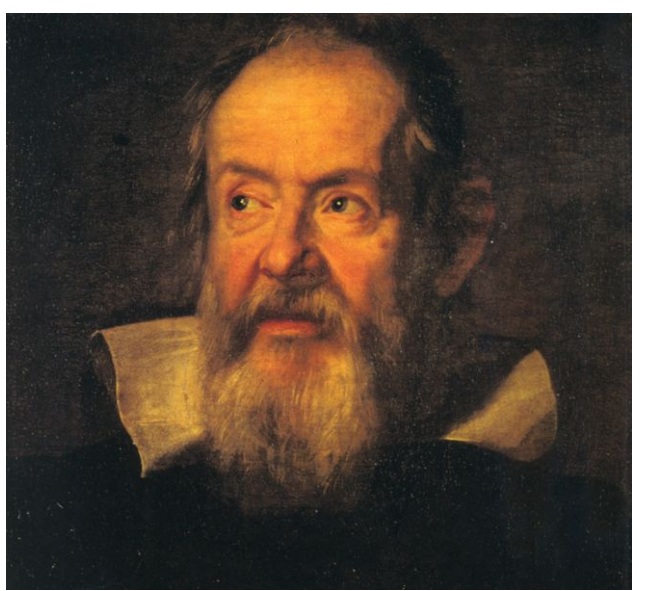

Figure 3: One of Sustermans' portaits of Galileo.

explained the behaviour of bodies and their natural positions of rest. Galileo took this notion and applied it to bodies in motion. His monumental postulation was that buoyancy, in addition to determining a body's position of rest, furthermore determines how fast a body will reach that position of rest. He used this force of buoyancy to try to explain why objects fell at the speed they did.

It is wrong to say that he devised a dynamical law based on static principles. His theorems are a generalisation of Archimedes' static principles, which are then derivable from Galileo's: the converse is untrue.

These notions were not wholly new. Instead of using a ratio of weight to resistance in order to explain motion, Galileo described it as a natural motion from which was subtracted the effect of the medium. Instead of having velocity determined by the ratio of a body's weight to the medium's resistance, it was to be determined by a natural value minus some part due to the resistance of the medium. The approach, ingenious though it was, led to no hoped for grand principle.

The comparison between Galileo and Avempace is commonly drawn, as Avempace had postulated the same kind of thing: discarding the Aristotelian ratio. Galileo was certainly aware of Avempace's work, through what Averröes wrote of it $^{12}$. It is unfair to say that Avempace was the originator of this sort of analysis, as it predates him by hundreds of years. This theory again goes back to John Philoponus, who was also well known to Galileo. Additionally, Avempace did not postulate Galileo's explanations for the causes of motion (i.e., a dynamic buoyancy law).

A note should be made on his supposed discovery

\footnotetext{
${ }^{12}$ In his early notes, Galileo cites Averröes ahead of all, save Aquinas. See the essay "Galileo and the Doctores Parisienses" in (Wallace, 1981).
}

of the equality of fall-rates. Galileo did not make this discovery. The story is that in 1589 he dropped various cannon balls from the Leaning Tower of Pisa, and thus the world came to know that all objects fall at the same rate. The story is wrong on several counts. Firstly, this experiment does not even demonstrate equal fall rates: it only shows that objects of the same composition fall at the same rate, independent of their weight. Secondly, in 1589, Galileo did not believe in the equality of fall-rates. His notes from this period (Galileo, 1590) state that objects of equal density fall at the same rate, but that denser objects fall faster than less dense ones:

I say therefore that in a vacuum, heavier bodies would descend more rapidly than lighter ones, because the excess of the heavier bodies over the medium would be greater than the excess of the lighter ones.

That bodies of the same density fell at the same rate had been stated already by Bradwardine two hundred years before. Thirdly, the selfsame experiment had already been performed by Giambattista Benedetti years before, and his work was known to Galileo.

It is difficult to ascertain when Galileo concluded that all objects fall at the same rate. He withheld publication on this subject for many years. The initial cause of his withholding was his own desire to bring the subject to a completion before revealing it. The later cause was the restrictions placed on him by the Inquisition. He knew of it by 1604 , as he revealed it in correspondence with a confidant. He felt betrayed when a friend of his mistakenly revealed it to the world in the early 1630s, and only published anything on the matter towards the end of his life.

For all Galileo's effort, he never satisfied himself with his explanations of the causes of motion. In Dialogues Concerning Two New Sciences (Galileo, 1638), towards the close of his life, he sadly confessed to this failure. After listening to the thoughts on dynamics voiced by Simplicio and Sagredo, Salviati, proponent of Galileo's philosophy, makes the comment:

Now, all these fantasies, and others too, ought to be examined; but it is really not worth while. At present it is the purpose of our Author merely to investigate and to demonstrate some of the properties of accelerated motion (whatever the cause of this acceleration may be)...

Here Galileo resigns himself to never finding what he sought. His characters instead progress through thorough discussions of the kinematics of motion alone: 
Salviati and Sagredo dragging Simplicio ${ }^{13}$ by the coattails into modern science:

...we have decided to consider the phenomena of bodies falling with an acceleration such as actually occurs in nature and to make this definition of accelerated motion exhibit the essential features of observed accelerated motions.

Over the course of the rest of the book, Galileo sets forth his definitions of uniform and accelerated motion in lightning fast demonstrations. The topics of discussion then go through motions of various things, especially that of projectiles. This work sounded the death knell of Aristotle's physics. In discussing a body thrown upwards, then falling back downwards, Simplicio voices the two thousand year old distinction between natural and violent motion. Sagredo replies:

...this distinction between cases which you make [i.e., violent and natural] is superfluous or rather non-existent.

Much is said about Newton unifying motion in the heavens and motion on Earth: that is, recognising that the laws apply equally so to the orbit of a massive planet as they do to the fall of a tiny apple. Very little is said about unifying natural and violent terrestrial motion, and yet it took two millennia of thought before the two were recognised as one and the same. The separation of the two had permeated Western science for two thousand years, and it was Galileo who demonstrated the insight to finally and permanently demolish this concept.

His dynamics were noteworthy and had a very large influence over his successors, but the true contribution of Galileo is in his kinematics, not in his dynamics. He could explain what he observed: uniformly accelerated motion, but he could not explain the causes behind it. While he failed, he set a great example for his successors: one that was well learnt and will never be forgotten. His dynamics were laden with no mystical indistinct properties. They were laden with definitions and analyses.

Aristotelian dynamics had been staggered by many deserved blows, but had kept standing, in various poses, for two thousand years. Galileo delivered the coup de grâce, putting it to its long overdue rest.

By the mid 17th century, dynamics was understood in the murkiest of ways. A myriad of problems, each requiring its own ingenious solution, was solvable almost solely by special cases. Much of this problem solving

\footnotetext{
${ }^{13}$ The name is a portmanteau of Simplicius, the classical Aristotelian, and the word for simpleton.
}

was needed before any general laws of dynamics could finally be grasped. Today we learn the principles, and then how to apply them. The developers of the field solved extraordinarily difficult problems, and lots of them, before any true governing principles were found. We learn the principles and then tackle problems, but the physicists had to tackle the problems before they could see the principles.

The giant of mechanics in the years leading up to Newton was the Dutchman, Christiaan Huygens (1629-1695). He was the first to explain oscillations of a finite pendulum, which he did so for a special case. His writings on solid body collisions had a monumental effect on the world. He observed that after two solid bodies collide, their collective speed may well be increased or decreased, but their collective momentum remains the same: perhaps the first true expression of the conservation of momentum ${ }^{14}$. He furthermore recognised that in rigid body collisions, the centre of gravity of the system remains in uniform motion: a hugely penetrating notion.

\section{Sir Isaac Newton}

Sir Isaac Newton (1643-1728), depicted in Figure 4, made contributions to virtually every area of natural philosophy, mathematics, optics and astronomy. His monumental publication, Philosophe Naturalis Principia Mathematica, usually called The Principia in short, was published in 1687. It is likely the most influential book in the field of classical mechanics, yet is little read. Its purpose was set forth in its preface:

...mechanics will be the science of motion resulting from any forces whatsoever, and of the forces required to produce any motion...

Newton set out to explain phenomena throughout the universe. What lay within was to apply everywhere, and to every process. The trajectory taken by a cannon ball was to be governed by the same laws which governed the orbits of the planets.

As the start of his work, he states his definitions of mass, momentum, inertia and forces, both through contact and at a distance. He then states his laws:

First Law Every body perseveres in its state of rest, or of uniform motion in a right line, unless it be compelled to change that state by forces impressed upon it thereon.

\footnotetext{
${ }^{14}$ Descartes is often credited with this concept, but his notion was the bulk of an object times its scalar speed. His rationale was entirely different too.
} 


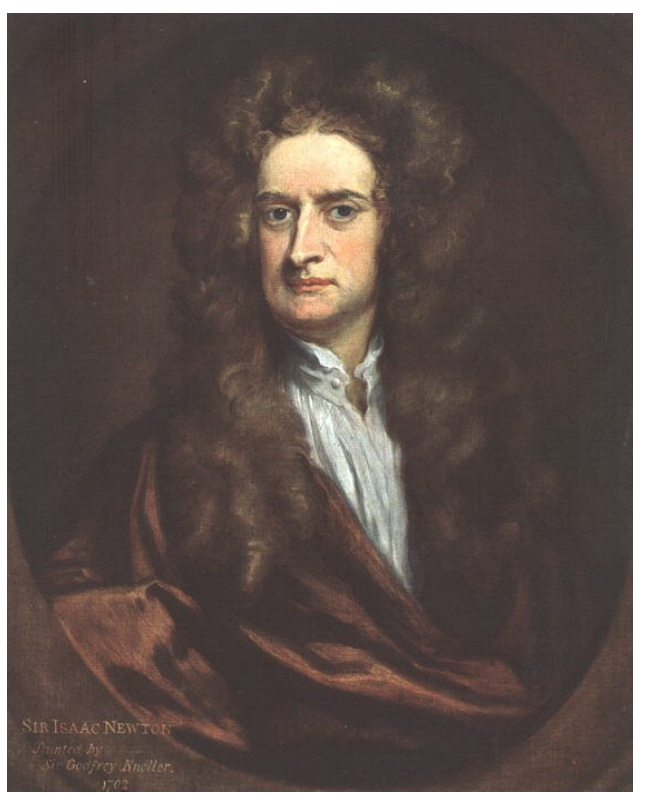

Figure 4: One of Kneller's portaits of Newton.

Second Law The alteration of motion is proportional to the motive force impressed; and is made in the direction of the right line in which that force is impressed.

Third Law To every action there is always opposed an equal reaction: or the mutual actions of two bodies upon each other are always equal, and directed to contrary parts.

It is broadly divided into three books, each of which alone would eclipse almost any other. Books One and Two are titled Of the Motion of Bodies, being split into two exhaustive analyses. The third is titled The System of the World.

The first book analyses motions in a void. From his laws, he analyses a multitude of motions, such as elliptic, parabolic and hyperbolic orbits around some focus. He investigates the forces that maintain these, i.e. the centripetal forces. Universal gravitation is introduced. After showing how point masses behave in the void under gravitation, he demonstrates that finite bodies can be treated as such. Kepler's Laws follow directly.

The first book organised and systematised principles, some of which were at least dimly understood before, but these principles had never been organised together into a system of analysis for application everywhere.

The second book sets out to explain motion on Earth, where motion does not occur in a void: he sought the details of motion in resisting media. It is here that Newton departs from his program of deducing physical behaviour based on his laws: he finds but little use for them. For example, in all his treatments of fluidic motion he finds no room to apply his principle of momentum. In contrast, he conjures ingenious hypotheses to explain a myriad of things ranging from projectile motions to the speed of sound in air. This book is a testament to Newton's towering stature as a mathematician and dynamicist. The second book of the Principia is almost entirely new. The scholium of the first section of it reads:

But, yet, that the resistance of bodies is in the ratio of the velocity, is more a mathematical hypothesis than a physical one.

This sentiment is applicable to much of the hypotheses in the book. Today it is mostly forgotten. The book is dominated by hypothesis after hypothesis, with Newton displaying his flair for creative solutions: often precise, often an excellent approximation, but also often wrong and today of only historical value.

There are veins of gold hidden within. His observation that fluidic resistance is proportional to the square of velocity can be found, as can the first description of internal fluidic friction:

The resistance arising from the want of lubricity in the parts of a fluid is, cæteris paribus, proportional to the velocity with which the parts of the fluid are separated from each other.

That most of the results were incorrect cannot be a criticism of Newton either as a physicist or mathematician. The contribution of this book is immeasurable. For instance, it constitutes the beginning of fluid dynamics, and studied many of its problems for the first time. From his efforts, his contemporaries and successors were gifted with a bridgehead from which to attack these subjects in earnest. A myriad of potential motions through fluids is contemplated. The book is the staging point for hydrodynamics. Newton contemplated which hullform might pass through the water with least resistance, introducing an optimisation problem that found application throughout the 19th century.

The third book set forth his solutions to problems in celestial dynamics, with great success. Kepler's Laws of Planetary Motion had resulted from Newton's own, and he performed exhaustive analyses of the Solar System.

The deficiencies in the Principia are little discussed. To the modern scholar, it is often impenetrable and confusing; the language of mathematics having evolved so much since then. A common remark made about the Principia is that Newton strangely resorts to geometrical methods instead of his own calculus. Newton does 
not use his notation of fluxions, but even as soon as we arrive at Lemma II of Book I, the notion of calculus is present, if in an unfamiliar form.

For rigid body mechanics, there is no treatment of rotation. Although Newton says that the spinning top "does not stop spinning except insofar as it is slowed by air." there is no justification given. His statement appears directly after his statement of the First Law, but this law cannot tell us anything of the spinning top. Newton might have perceived that the top continues to spin, just as it would continue in linear motion if so impelled, but it is not possible to explain the spinning top using what is within the Principia. There is certainly no treatment of angular momentum. The motion of a rigid body cannot be described by the methods given in the Principia.

There is no treatment of flexible bodies, such as the catenary curve or the vibrating string, nor is there any analysis of the finite body pendulum. No equations of motion appear for systems of more than two free masses, or one constrained. A prime example of the field's infancy is the three-body problem. Newton attempted to solve this problem, but the contents of the Principia are insufficient to do so. He devised insightful approximations and valid inequalities, but the threebody problem was insoluble from his principles. His talent in this area is evident, as his work would not be surpassed until the mid-18th century by the efforts of Euler and Lagrange.

That Newton did not solve all of mechanics' problems is not a criticism at all, but only part of a clearheaded appraisal of what he did do. His achievements were monumental. He ought not to be credited with the completion of classical mechanics, but rather its beginnings.

In the century following The Principia's publication, the field of mechanics swelled immensely. For all the credit given to Newton, the world ought to be equally grateful to his contemporaries and successors, especially Leonhard Euler, the Bernoullis Jakob and John, and Joseph Lagrange. These are the men who synthesised what we now apply today.

\section{Newton's Contemporaries and Successors}

If The Principia contains no treatment of angular momentum, contrary to popular belief, then where and when did this law arise and who discovered it? The answer is difficult to ascertain, as the principle was applied for many years before it was recognised for what it was. For a comprehensive analysis, see Whence the law of moment of momentum? in (Truesdell, 1968).

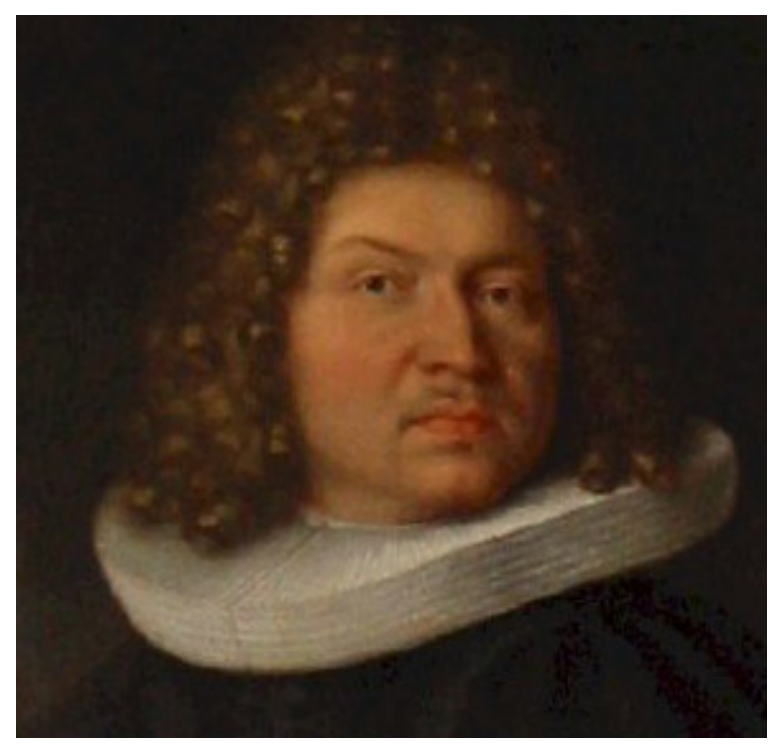

Figure 5: Jakob Bernoulli: towering mathematician and physicist of the 1700 s and early 1800 s.

The law of angular momentum is commonly treated as a consequence of linear momentum, but that is no more true than the common statement that Newton's First Law can be ascertained from his Second. This approach works for special cases only: it is not true in general. The Newtonian equations cannot contemplate deformable bodies or motion of a continuum without severe restriction. Angular momentum is a law of mechanics independent from any other. It took most of the 18th century for this to be realised.

In the Acta Eruditorum of 1686, Jakob Bernoulli (1654-1705), shown in Figure 5, analysed the motion of a pendulum using the ancient law of the lever: i.e., by balancing the moments. By applying this static problem to the dynamic problem of the pendulum, he sought a new methodology for mechanics. His attempt at this stage was flawed, but was published in correct form in 1703 (Bernoulli, 1703). His efforts led him to introduce many concepts which are now elementary, or even obvious, today.

By balancing the moments, the law of the lever is found. This static equilibrium condition was generalised into a dynamic equilibrium. Jakob Bernoulli wrote that the force of the lever can be regarded as equivalent to the acceleration per unit mass reversed in sign, thereby restoring a static condition. His was the earliest proper explanation of inertial force, and is a progenitor of what is now by convention called D'Alembert's Principle.

Jakob Bernoulli's statement of the moment of momentum can not be considered a consequence of apply- 
ing Newton's Second Law. Its first appearance, flawed though it was, predates the publication of the Principia by a year. To emphasise: the appearance of the law governing angular motion predates Newton's 2nd law, which governs only linear motion. That said, it is only to be found and understood with great difficulty. The impenetrability of Jakob Bernoulli's work is evident, since it took many years before the genius within was recognised and developed by Euler.

Through analysing the catenary curve ${ }^{15}$, it was Jakob Bernoulli who first recognised that solutions could be derived by balancing forces applied to infinitesimal portions of the cable, and furthermore that the selfsame solutions could be derived by balancing the moments acting on those infinitesimal portions: two essential principles of mechanics are equivalent for certain systems. This apparent equivalency caused the great physicists of the 18th century to seek, in vain, the single unifying principle through which all of mechanics could be analysed.

Using this work on the catenary as a springboard, Jakob Bernoulli vaulted into an analysis of elastic beams: the bending of finite bodies, which he published in the Acta Eruditorum of 1694. Here he recognised that the balancing of forces or moments alone was insufficient to the task. Between each infinitesimal stretch, there must be a contact force and moment.

In addition to these mammoth contributions, Jakob Bernoulli was the first to state how the motion of a constrained system can be analysed. Given the constraints, propose the forces which maintain these constraints. The motion of a system of constrained masses can then be analysed. Seemingly obvious today, the idea finds no ancestor before Jakob Bernoulli.

If it is typical to elevate some beyond their true achievements, it is equally typical to undermine those with achievements beyond measure. The laws, equations and principles named after Leonhard Euler (1707-1783) devastate Stigler's Law of Eponymy ${ }^{16}$, and yet he is the very reason that the law applies virtually everywhere else. It is through Euler that much of dynamics was delivered to the modern world.

Between 1747 and 1750, Euler took his own works on constrained systems, and applied them to the threebody problem. In this work, he wrote:

The foundation... is nothing else than the known principle of mechanics, $d u=p d t$, ...we can see that this principle holds equally for each partial motion into which the true motion is thought of as reduced.

\footnotetext{
${ }^{15}$ The shape that a thin hanging cable assumes under its own weight.

16 "No scientific discovery is named after its original discoverer."
}

Euler is here saying that what had been found was an approach that applied to any dynamic process, and that it additionally applied to every part of that process. It was simply not understood prior to Euler's paper. In retrospect it seems too obvious to even mention. This retrospection emphasises the difficulty of analysing the history of science: today, it is thoroughly difficult not to see this principle as self-evident in Newton's writings. What is obvious to us today is supposed to have been obvious then, but that is wrong.

In Euler's paper, Discovery of a New Principle of Mechanics (Euler, 1750), he wrote down the following:

$$
F_{x}=M a_{x}, \quad F_{y}=M a_{y}, \quad F_{z}=M a_{z} .
$$

Following his statement of the new principle, he derived the tensor of inertia by taking the moments about the centre of gravity. By these equations, Euler claimed, all mechanical problems could be solved. That we now call these equations Newton's Second Law is immaterial. In the words of Truesdell:

...they occur nowhere in the work of Newton or of anyone else prior to 1747 . It is true that we, today, can easily read them into Newton's words, but we do so by hindsight.

Although Euler initially believed that the issue was resolved, he shortly came to realise his mistake. The principle of angular momentum lay hidden. The full classical equations would not be written down for another two decades.

The rotations of even a rigid body were problematic, let alone of a system of particles or continuum. That a rigid body could rotate ad infinitum was dimly perceived for almost a century before it was properly explained. As mentioned, Newton's spinning top is a key example, but we simply cannot admit stabs-inthe-dark. Throughout the early to mid 18th century, physicists had been unable to explain rotational motion in more than a single axis.

Euler contemplated the problem in the early 1730s, but did not approach it again for another decade. The driving force was his work on naval architecture in Scientia Navalis (Euler, 1749). Here he was forced to deal with oscillations very different from the simple planar type. He hypothesised that each body has three orthogonal axes about which it may rotate.

The first rigorous treatment of these axes was by the Hungarian physicist, Ján Andrej Segner (1704-1777). He proved that free rotation is possible through a minimum of three individual axes, there being more than three for special cases of symmetry (spheres, etc.). Euler recognised the strength of Segner's reasoning, and was the first to reason that these axes all had to pass through the centre of gravity. 
1776 saw the birth of a foundation of classical mechanics. It was in this year that Euler published his First and Second Axioms (Euler, 1776):

$$
\begin{aligned}
& \mathbf{F}=\dot{\mathbf{P}}, \quad \mathbf{P}=\mathbf{M} \mathbf{v} \\
& \mathbf{L}=\dot{\mathbf{H}}, \quad \mathbf{H}=\mathbf{I} \omega .
\end{aligned}
$$

At last, the road devised by Newton, and hewn by many, had been paved by Euler. The laws of vectorial mechanics were understood and formulated then just as they are today.

\section{The Indirect Approach}

Newton's approach takes force and momentum as its basis. It is often called the direct approach, or vectorial dynamics. D'Alembert wrote what is anything but a shining endorsement of the direct approach in his book Treatise on Dynamics in 1743:

Why should we appeal to that principle used by everybody nowadays, that the accelerating or retarding force is proportional to the element [i.e. differential] of velocity, a principle resting only on that vague and obscure axiom that the effect is proportional to the cause? ...we shall be content to remark that the principle, be it true or be it dubious, be it clear or be it obscure, is useless to mechanics and ought therefore to be banished from it.

The lionisation of the Newtonian approach by the British certainly was not quite mirrored everywhere on the continent. In contrast to the direct approach, and with equal validity, the scalar quantities of energy and work can serve as a basis for an approach called the indirect approach, or analytical dynamics (Williams Jr., 1996).

The history of analytical dynamics is just as cloudy and obscure as the development of Newton's Laws. Gottfried Wilhelm Leibniz (1647-1716) is the earliest true standard bearer for using energy and work as the bases for mechanical principles. He posited that through any process, a vis viva (living force) is preserved. This term he equated to $m v^{2}$ and so Leibniz's living force is just twice the kinetic energy. He believed, and so did his contemporaries, that conservation of vis viva contradicted the Cartesian and Newtonian notion of conservation of momentum. Leibniz wished to use this vis viva, along with his dead force (potential energy), as the basic principles of mechanics. This "living" force irritated the delicate sensibilities of many, and with good reason. A living force seemed to invite the teleological and theological qualities of the ancient sciences to return from the dead.

One must question why Leibniz objected so much to the Cartesians' and Newtonians' use of momentum. For them, landmark results were already inbound using the principle of momentum and its conservation. What was special about this vis viva, and why did velocity appear twice? These notions stem from Galileo's Two New Sciences.

In his final Dialogue (Galileo, 1638), Galileo made key observations related to the indirect approach. Falling from a given height, a body acquires a velocity that is precisely the same velocity required to raise the body back to the given height. Since the square of the velocity acquired is proportional to the height, it seemed reasonable to surmise that $v^{2}$ has a link to some fundamental property of motion. Running along the same vein of gold, he noted that velocity acquired by a body rolling down an incline is only influenced by the height of the fall and not by the inclination itself: he recognised that the velocity was independent of the path.

Leibniz considered many cases using his live and dead forces. Indeed, many problems can be solved by examining the energy quantities at key points. Instead of the Newtonian momentum, and its alteration through impressed forces, Leibniz considered kinetic energy, and its alteration through the work done by impressed forces. The mathematics behind what lay ahead was undeveloped in his day, and the true leap forward for the indirect approach would have to wait many years from Leibniz, until it found its home with Euler and Lagrange. Before the indirect method could come to fruition, the calculus of variations was required.

Along those lines, an interesting period in this development is the brachistochrone challenge of the late 17 th century. Brachistochrone is a portmanteau of brachistos and chronos: Greek for shortest and time respectively. The problem is to take a bead on a frictionless wire, acted upon only by gravity, and to then determine the quickest possible route between two points $\mathrm{A}$ and B. It can be seen in Figure 6 . The first version of the problem was posed by Galileo in Two New Sciences (Galileo, 1638), but his solution was incorrect, mistakenly thinking that the solution was the arc of a circle. He at least recognised that it was not a straight line.

The first person to solve the problem was John Bernoulli. He posed this problem to the mathematicians of Europe as a challenge in the Acta Eruditorum of 1696 .

In choosing the wording of his challenge, Bernoulli gave an unmistakable hint at how he had solved the 


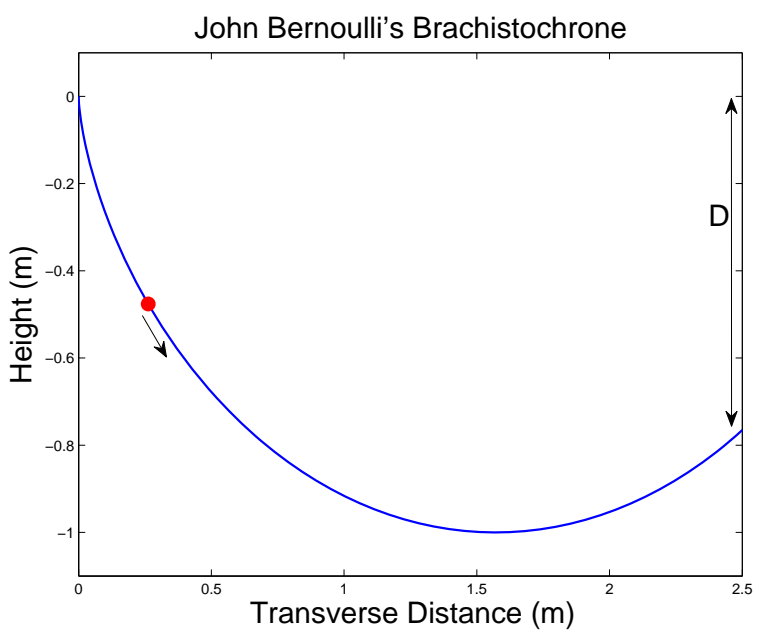

Figure 6: John Bernoulli's Brachistochrone.

problem. He invoked both Pascal and Fermat. Pascal had offered prizes for challenges on the cycloid four decades prior, while Fermat showed that light always takes the path of least time (Fermat's Principle). The solution to John Bernoulli's challenge was the former's curve, and his method was by applying Fermat's principle.

Solutions soon arrived from his elder brother Jakob, Leibniz, de L'Hôpital and an anonymous one from Newton ${ }^{17}$; all showing the solution to be the cycloid.

This challenge led to a clash between the fragile egos of the two Bernoullis. Following publication of the solutions to the brachistochrone problem in 1697, Jakob Bernoulli posed a more difficult version, again in the Acta Eruditorum. The first version sought the minimum time to a certain point. Jakob Bernoulli instead posed a problem to minimise the travel time to a vertical line. That is, to find out which of all the possible cycloids reaches the line first. The reposed problem is depicted in Figure 7.

This problem was quickly tackled by both Bernoullis, Leibniz and Euler. The answer to the problem is the cycloid which passes through the line at a right angle to it. It is not, however, the answer to the problem which makes this challenge especially noteworthy.

The second version of the brachistochrone was obviously another minimum time problem, but now it was a problem to be solved by contemplating all possible paths. It was hardly the first optimisation problem to

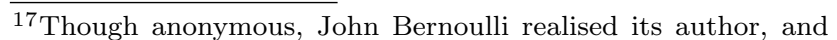
remarked in a letter that "we know indubitably that the author is the celebrated $\mathrm{Mr}$ Newton; and, besides, it were enough to understand so by this sample, as ex ungue Leonem."(Whiteside, 2008). The phrase is usually given incorrectly as "tanquam ex ungue leonem."
}

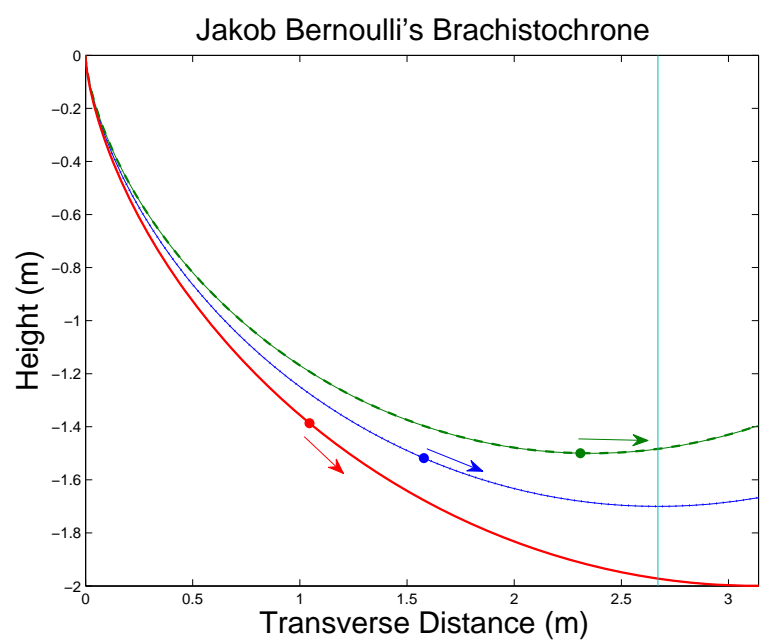

Figure 7: Jakob Bernoulli's Brachistochrone.

be contemplated, but it was the particular one which, in being solved, led to the development of the calculus of variations. In the years of the early 18th century, the calculus of variations was formalised and organised by the likes of John Bernoulli, D'Alembert and Euler.

It was during this timeframe that D'Alembert and Euler finally generalised Jakob Bernoulli's inertial force. They formalised the pre-existing principle and showed that the principle of virtual work applied equally to bodies in motion. Mechanics was then gifted with a single variational principle.

The furtherance of the attempts to use energy and work to ascertain physical principles is due to the work of the Frenchman, Pierre de Maupertuis (1698-1759). He posited that in many processes, an action is minimised, with this action being defined according to the process. His perceptive qualities were remarkable. Most of his definitions of action found no use, but others did. For light, he posited in a 1744 paper, Agreement of several natural laws that had hitherto seemed to be incompatible, that this action was the integral of the speed over the path taken. With this principle, he struck gold, deriving Snell's Law from an indirect approach. His genius here mirrors what John Bernoulli did in his brachistochrone solution.

This principle of least action became ubiquitous throughout much of mechanics. In the same year, Euler published his own results on the matter. He posited it in a far more general and accurate way, and applied the integral of the momentum of a body over its path travelled, giving the reduced action, i.e., the action with kinetic energy alone. In the following years, he applied the same to static problems, taking variations of the potential energy. This method lead him to the classical result that a body, or a system of bodies, at rest 
always lie at a minimum of potential energy.

The thrust in this direction was taken up by the French-Italian mathematician, Joseph Lagrange (1736-1813). A contemporary of Euler in his later years and common collaborator, the two formulated the Euler-Lagrange equation, bringing the formulation of the indirect method on by leaps and bounds. Lagrange was the true champion of this method throughout his Mècanique Analytique (Lagrange, 1788). In addition, he recognised the usefulness of generalised coordinates, giving the method a towering strength: the invariance of the method to coordinate changes.

The future development of this field through Hamilton and Jacobi is rather well understood, and so here is a good point to close this history.

\section{Conclusion}

This article summed up, as concisely as the author could achieve, the development of the broad field of dynamics, progressing speedily from Aristotle, through Philoponus, on to Galileo, and Newton, and finishing in the details of how the great minds of the 18th century synthesised many of the modern principles of mechanics that still serve humanity well today.

The deficiencies of this article are numerous. It is perhaps placed in too smooth a narrative, implying the development of dynamics occurred along a single timeline, but that is emphatically not the case. For example, although the article transitions from Galileo to Newton, it is wholly unclear if Newton benefited from Galileo's work. Were the essay rather to have looked to Newton's inspiration, it would more likely have looked back to Apollonius of Perga, and how his works were used by Newton. Such an article would be of a wholly different nature to the one presented here, which attempted to bring a short and reasonably concise history to a new audience.

\section{References}

Aristotle. Physics. Kessinger Publishing Co., 2004 edition, 330 B.C.

Bernoulli, J. Démonstration générale du centre de balancement ou d'oscillation, tirée de la nature du levier. Mémoires de l'Academie Royale des sciences, 1703. pages $78-84$.

Duhem, P. Research on the history of physical theories. Synthese, 1917. 83(2):189-200. doi:10.1007/BF00413756. Reprinted May 1990.

Euler, L. Scientia Navalis. Birkhäuser Basel, 1749. Written ca. 1738, edited by C. Truesdell.
Euler, L. Discovery of a new principle of mechanics. Mémoires de l'Academie Royale des sciences, 1750. Par. XXIII:196.

Euler, L. A new method for generating the motion of a rigid body (nova methodus motum corporum rigidorum degerminandi). Novi Commentarii academiae scientiarum Petropolitanae, 1776. 20:208-238. E479.

Galileo. De Motu. University of Wisconsin Press, 1590. Trans. S. Drake., 1960.

Galileo. Dialogue Concerning Two New Sciences. Elsevier, 1638.

Lagrange, J. L. Mécanique Analytique (Analytical Mechanics). Boston Studies in the Philosophy of Science, 2001 ed. Springer-Verlag, 1788.

Mach, E. Die Mechanik in ihrer Entwicklung: Historisch-kritisch dargestellt. 1886.

Moody, E. Studies in Medieval Philosophy, Science, and Logic: Collected Papers, 1933-1969, chapter 5, pages 189-201. University of California Press, 1975.

Newton, I. Philosopha Naturalis Principia Mathematica. Prometheus Books, 3rd ed. 1995 edition, 1687. Translated by Andrew Motte.

Noll, W. On the past and future of natural philosophy. Journal of Elasticity, 1996. 84(1):1-11. doi:10.1007/s10659-006-9068-y.

Philoponus, J. Ancient Commentators on Aristotle, chapter On Aristotle's Physics. Gerald Duckworth \& Co. Ltd., 2006.

Truesdell, C. Essays in the history of mechanics. Springer-Verlag, Berlin, Germany, 1968.

Wallace, W. A. Prelude to Galileo. D. Reidel Publishing Company, 1981.

Whiteside, D., editor. The Mathematical Papers of Isaac Newton: 1697-1722. Cambridge University Press, 2008. Page 9.

Williams Jr., J. H. Fundamentals of Applied Dynamics. John Wiley \& Sons, Inc., 1996.

Zimmerman, F. Philoponus and the Rejection of Aristotelian Science, chapter Philoponus' Impetus Theory in the Arabic Tradition, pages 121-129. Cornell University Press, 1987. 